\title{
ANNI - A pulsed cold neutron beam facility for particle physics at the ESS
}

\author{
Torsten Soldner ${ }^{1, a}$, Hartmut Abele ${ }^{2}$, Gertrud Konrad $^{3,2}$, Bastian Märkisch $^{4}$, Florian M. Piegsa ${ }^{5}$, Ulrich Schmidt $^{6}$, \\ Camille Theroine ${ }^{1,4,7}$, and Pablo Torres Sánchez $z^{1,8}$ \\ ${ }^{1}$ Institut Laue-Langevin, CS 20156, 38042 Grenoble Cedex 9, France \\ 2 Atominstitut, TU Wien, Stadionallee 2, 1020 Wien, Austria \\ ${ }^{3}$ Stefan Meyer Institute for Subatomic Physics, Austrian Academy of Sciences, Boltzmanngasse 3, 1090 Wien, Austria \\ ${ }^{4}$ Physik-Department ENE, Technische Universität München, James-Franck-Str. 1, 85748 Garching, Germany \\ ${ }^{5}$ Laboratory for High Energy Physics and Albert Einstein Center for Fundamental Physics, University of Bern, Sidlerstrasse 5, \\ 3012 Bern, Switzerland \\ ${ }^{6}$ Physikalisches Institut, Universität Heidelberg, Im Neuenheimer Feld 226, 69120 Heidelberg, Germany \\ ${ }^{7}$ European Spallation Source, Box 176, 22100 Lund, Sweden \\ ${ }^{8}$ Fac Ciencias, Universidad de Granada, Av. Fuente Nueva S/N, 18071 Granada, Spain
}

\begin{abstract}
Pulsed beams have tremendous advantages for precision experiments with cold neutrons. In order to minimise and measure systematic effects, they are used at continuous sources in spite of the related substantial decrease in intensity. At the European Spallation Source ESS these experiments will profit from the pulse structure of the source and its 50 times higher peak brightness compared to the most intense reactor facilities, making novel concepts feasible. Therefore, the cold neutron beam facility for particle physics ANNI was proposed as part of the ESS instrument suite. The proposed design has been re-optimised to take into account the present ESS cold moderator layout. We present design considerations, the optimised instrument parameters and performance, and expected gain factors for several reference experiments.
\end{abstract}

\section{Introduction}

Neutron particle physics is part of the precision frontier in particle physics. New particles and interactions are not observed directly but detected by their contributions to observables at low energy. In this way, experiments with neutrons can probe new physics at the multi$\mathrm{TeV}$ scale, complementary to and competitive with searches at colliders [1-6]. Precision is key in this endeavour. Experimental progress requires high statistics and an adequate control of systematic effects. Important experimental conditions can be controlled by using pulsed beams:

Neutron velocity (energy, wavelength): The arrival time of a neutron at a given distance from the creation of the pulse indicates its velocity. This can be exploited to separate effects with different dependencies on the neutron velocity. Examples are the beam polarisation and transmission by a spin filter cell which can be used for precise polarimetry [7] or the induced magnetic field $\propto \mathbf{v} \times \mathbf{E}$ in the rest frame of a neutron moving in an electric field which is relevant for measurements of the neutron electric dipole moment (EDM) [8]. In a novel neutron interferometric concept for a precision measurement of the electric charge of the neutron the timing information is used to select neutrons of specific velocities [9]. Furthermore, parameters of neutron-velocity dependent optical elements can be

a e-mail: soldner@ill.fr varied to fit the neutrons arriving at a certain moment (time-dependent neutron optics). Examples are the amplitude of the oscillatory fields in $\pi / 2$ spin-flip coils enabling a $\pi / 2$ flip for all neutrons [10] or the opening of a disk chopper effectively working as beam monochromator.

Localisation of neutron pulse in time: At a pulsed beam the signal arrives within a short time span which increases the signal-to-background ratio compared to a continuous beam of the same time-averaged intensity, assuming similar levels of environmental background. Furthermore, signal and background are measured in time intervals that are very close to each other, allowing to subtract slowly-changing environmental background [11]. Beam-related background with a time constant large compared to the pulse duration (e.g. activation of windows or beta-active isotopes in a target, background from unwanted Penning traps in retardation spectrometers) is diluted compared to the prompt signal which increases the signal-tobackground ratio for beam-related background.

Localisation of neutron pulse in space: In contrast to a continuous beam, a neutron pulse can be observed without any contact to baffles or the beam stop thus preventing beam-related background, or fully inside a region with well-defined spectrometer response hence avoiding e.g. edge effects [11].

These conditions reduce or completely suppress many systematic effects. Therefore pulsed beams are used at 
continuous facilities in spite of the related substantial reduction in counting statistics. At a pulsed facility, these methods can be applied at full counting statistics. The ESS cold moderator will provide a 50 times higher peak brightness and a two times higher time-averaged brightness than the cold sources of the leading continuous facility (the high flux reactor of the Institut Laue-Langevin ILL) [12]. In spite of the substantially smaller viewed area of the ESS cold moderator, pulsed experiments at the ESS should gain more than one order of magnitude in counting rate compared to the ILL. This led us to propose ANNI [13], a pulsed cold neutron beam facility at the ESS optimised for precision measurements of neutron beta decay, hadronic weak interaction in calculable systems [14], and electromagnetic properties of the neutron.

\section{Design considerations}

The design of ANNI is driven by the goal to provide the maximum usable neutron flux as well as all advantages of pulsed beams. In order to provide unique velocity information, frame overlap must be absent for the accepted part of the spectrum. The wavelength band of $2 \AA$ to $8 \AA$ is chosen as reference as it includes $90 \%$ of the cold capture flux from the moderator. These requirements call for a short instrument which can be sensitive to background from the spallation source itself. Therefore, the next prompt pulse shall not fall inside a signal period at ANNI, i.e. arrive after the slowest selected neutrons have left the instrument. With the ESS repetition rate of $14 \mathrm{~Hz}$, the signal area of an experiment at ANNI should not surpass $34 \mathrm{~m}$ distance from the moderator. Note however that the usable signal area depends on the chosen wavelength band and that some experiments may be insensitive to background from the prompt pulse. Further arguments support the choice of a short instrument: The neutron pulse dilutes with distance from the moderator which makes its localisation in time less effective. The statistically optimal length of the beam line for a PERC-like spectrometer [15] is about $20 \mathrm{~m}$ (position of the last chopper) [16]. At about $22 \mathrm{~m}$ from the source, the instantaneous bandwidth (difference of longest and shortest wavelength present at a given distance and time) for the full source pulse falls below $0.5 \AA$ which enables a wavelength resolution of below $10 \%$ for cold neutrons. On the other hand, ANNI shall be versatile and be able to host long experiments such as the proposed BeamEDM [8] (length of the interaction area about $50 \mathrm{~m}$ ).

For pulse localisation the last chopper should be as close as possible to the experiment. In order to fit long spectrometers such as PERC [15] (8 $\mathrm{m}$ decay volume) in front of the $34 \mathrm{~m}$ argued above, the last chopper is placed at $26 \mathrm{~m}$ from the moderator. The ANNI guide ends at $22 \mathrm{~m}$, leaving $4 \mathrm{~m}$ space for beam preparation. Because of the high-energy particles created in the source, the ESS recommends that guide design excludes direct sight to the moderator at least twice (where subdividing walls in multi-channel benders are not taken into account), in order to prevent showers created in the first guide contact of these particles from reaching the experiment directly. The ANNI guide design follows this recommendation. This requires a strongly curved guide. The ESS moderator is flat which calls for a guide with smaller vertical than horizontal dimensions. Therefore the smallest guide curvature is achieved by curving in the vertical plane. In order to obtain a horizontal beam, an S-shaped geometry is used in the vertical plane.

Because of the small moderator, there is a delicate tradeoff between flux density and integral flux at the guide exit which was optimised using a suite of reference experiments. The resulting guide has about $10 \mathrm{~cm}$ height. In order to achieve a critical wavelength of about $2 \AA$ with economical supermirrors and a homogeneous beam profile in spite of the strong curvature, the curved sections are realised by multi-channel benders. The two benders have the same characteristic parameters $\gamma^{*}$ and $\lambda^{*}$ (defined in e.g. [17]) but were chosen to be different: The second is relatively compact, with a total length of $2.5 \mathrm{~m}$, in order to be able to replace it by a polarising bender. This allows for the highest polarised intensity if the requirements for the absolute polarisation are moderate. The instrument design also includes options for ultra-high polarisation.

ANNI's guide and chopper design was optimised for a selection of reference experiments covering the various beam configurations used at existing cold neutron beam facilities. These reference experiments were aSPECT [18, 19], NPDGamma [20,21], PERC [15] and PERKEO III [22]. Details on the selection of these experiments and the configurations used in the simulations are given in appendix A. The BeamEDM experiment [8] was taken into account for defining the spatial requirements of ANNI. The new projects BRAND [23] and HIBEAM [24] have expressed interest to measure at ANNI.

\section{Instrument layout}

\subsection{Simulations for guide optimisation}

The final simulations for the ANNI instrument proposal were performed in 2015 for the former $3 \mathrm{~cm}$ flat "Pancake" moderator design [25]. Technically, the simulations were performed with McStas [26] version 2.1 using the source component ESS_moderator (with sourcedef="2014" and the solid angle bug-fix from February 2015) and the guide component Guide_gravity. Each bender was composed of 16 straight guide elements with small gaps corresponding to a frequent practical realisation of long benders. Gravity was switched off in the simulations and geometrical guide imperfections were ignored. The reflectivities of guide supermirrors were described by realistic $m$-dependent parameters (where $m$ is the supermirror factor, example parameters for $m=3: \mathrm{RO}=0.99, \mathrm{Qc}=0.0219, \mathrm{~W}=0.0015, \mathrm{alpha}=2.74 \mathrm{in}$ McStas notation).

The final "Butterfly" moderator BF1 [12] has the same height of $3 \mathrm{~cm}$, but contains a thermal section. This reduces the effective width of the cold part to about $8 \mathrm{~cm}$ for beam port $\mathrm{E} 5$, the beam port provisionally allocated to ANNI [27], compared to about $17 \mathrm{~cm}$ of the "Pancake" moderator. Simulations for this moderator were performed with McStas version 2.4 and the source component ESS_butterfly; the beam port was specified by sector="E" and beamline=5. Each instrument can orient the guide to the thermal or the cold section of the moderator or opt for bispectral extraction [12]. For ANNI the cold section can be selected by starting the guide at $x=4.3 \mathrm{~cm}$ and aligning it parallel to the beam port axis or by starting the guide at $x=0$ and aligning it at $-1.26^{\circ}$ 


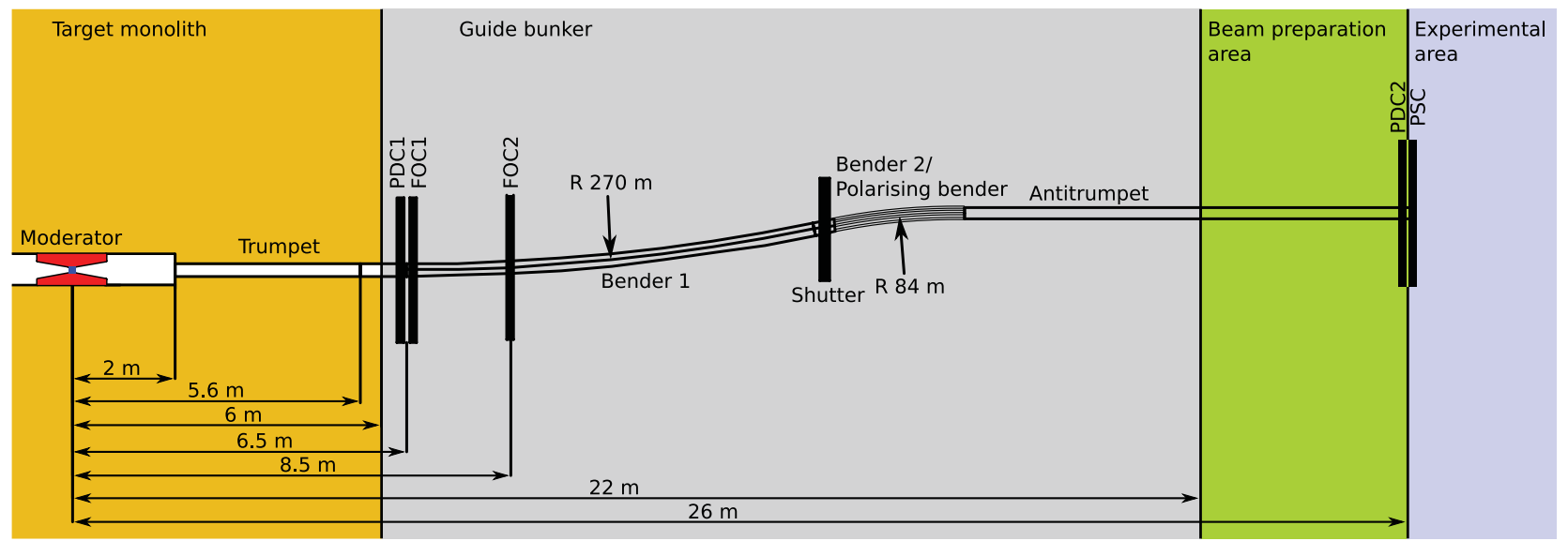

Figure 1. Side view of the ANNI beam line (schematic). The vertical scale is stretched by a factor of 4 for better readability. See text and Tables 1 and 2 for details.

Table 1. Key parameters of the guide components. Dist: Distance from the moderator to the start of the element. Rad: Radius of curvature. Negative means bend downwards. Len: Length of element. X-section: Width $\times$ Height. Trumpet and antitrumpet change the cross-section linearly from start to end (indicated by arrow). NOC: Number of channels in vertical direction. Channels are separated by $1 \mathrm{~mm}$ thick plates. $m$ value: Supermirror coating for all sides or separately for Left, Right, Top, and Bottom seen from the moderator. $\gamma^{*}$ and $\lambda^{*}$ : Characteristic parameters of curved guides.

\begin{tabular}{lcccccccc}
\hline Element & $\begin{array}{c}\text { Dist } \\
{[\mathrm{m}]}\end{array}$ & $\begin{array}{c}\text { Rad } \\
{[\mathrm{m}]}\end{array}$ & $\begin{array}{c}\text { Len } \\
{[\mathrm{m}]}\end{array}$ & $\begin{array}{c}\text { X-section } \\
{\left[\mathrm{cm}^{2}\right]}\end{array}$ & NOC & $m$ value & $\begin{array}{c}\gamma^{*} \\
{[\mathrm{mrad}]}\end{array}$ & $\begin{array}{c}\lambda^{*} \\
{[\AA]}\end{array}$ \\
\hline Trumpet & 2.0 & $\infty$ & 3.6 & $9 \times 6 \rightarrow 13 \times 6$ & 1 & LR: 3.5, TB: 3.0 & \\
Straight 1 & 5.6 & $\infty$ & 0.9 & $13 \times 6$ & 1 & 3.0 & & \\
Bender 1 & 6.5 & 270 & 8.0 & $13 \times 6$ & 2 & LRT: 3.0, B: 3.5 & 14.7 & 2.44 \\
Straight 2 & 14.5 & $\infty$ & 0.4 & $13 \times 6$ & 2 & 3.0 & & \\
Bender 2 or Po- & 6.5 & -84 & 2.5 & $13 \times 6$ & 6 & LRB: 3.0, T: 3.5 & 14.7 & 2.44 \\
larizing bender & & & & & & & \\
Antitrumpet & 17.4 & $\infty$ & 4.6 & $13 \times 6 \rightarrow 11 \times 7$ & 1 & LR: 3.5, TB: 3.0 & & \\
\hline
\end{tabular}

to the beam port axis (all in McStas coordinates). In practice, an intermediate solution may best fit the guide into the beam port; according to McStas simulations this has negligible influence on the performance. For this paper, only the horizontal guide dimensions were re-optimised whereas the vertical geometry was kept unchanged compared to the proposal [13]. The results presented here are based on the simulations for the Butterfly moderator. An ANNI McStas file is available with the arXiv version of this paper [28].

\subsection{Guide layout}

A vertical cut through the ANNI guide is shown in Fig. 1. Table 1 lists the key parameters of all guide components.

As illustration of the optimisation procedure, Fig. 2 shows the selection of the central, entrance and exit width of the guide from the simulations with the Butterfly moderator. The optimisation in the vertical plane is more complex since the curvature has to be taken into account. According to the simulations it is preferable to expand the guide from $6 \mathrm{~cm}$ to $7 \mathrm{~cm}$ height only at the end, after the curved section, which is counter-intuitive in terms of ballistic transport but allows for larger radii of curvature.

\subsection{Floor plan}

The proposed floor plan is shown in Fig. 3.

The guide ends at $22 \mathrm{~m}$ from the moderator and is followed by a $4 \mathrm{~m}$ long beam preparation area where neutron optical elements can be installed to shape the beam for a specific experiment. This includes additional polarisers, flippers, focussing optics, straight guides to transport the full flux and collimation systems. The beam preparation area shall be protected by a casemate but accessible if the shutter is closed.

The experimental area of ANNI starts at $26 \mathrm{~m}$ from the center of the moderator. We propose a width of $5 \mathrm{~m}$ for the experimental area, which corresponds to $\pm 5.5^{\circ}$ at $26 \mathrm{~m}$. The angle between beam ports being $6^{\circ}$, the left and right neighbor beam ports should not be allocated (or their instruments sufficiently downstream). The experimental area should be $25 \mathrm{~m}$ long. We propose to reserve the full angular sector of $\pm 6^{\circ}$ between the neighbor beam ports for ANNI as this would allow to prepare and commission the next experiment close to the measuring position, reducing time for changeovers. In order to realise the BeamEDM experiment [8], the experimental area should be extendable to $50 \mathrm{~m}$ length which is possible at the provisionally allocated beam port E5 [27]. 


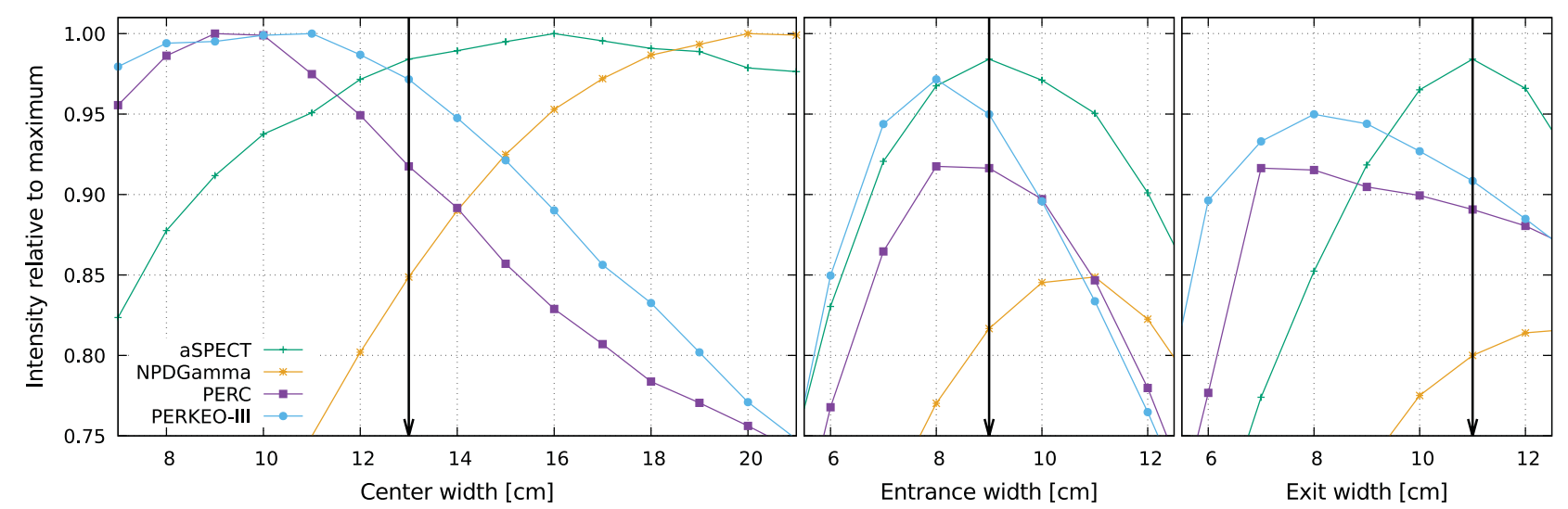

Figure 2. Optimisation of central, exit and entrance width of the guide system. All other guide settings were fixed to the values given in Table 1. Intensities are plotted relative to the maximum for the respective reference experiment (see appendix A) in the full parameter space. In the left plot, the maximum intensities for a given central width and free entrance and exit widths are plotted. The arrow indicates the selected central width $(13 \mathrm{~cm})$ which is then fixed for the other two plots. In the middle plot, the maximum intensities for a given entrance width and free exit width are plotted; the arrow indicates the selected entrance width $(9 \mathrm{~cm})$ which is then fixed for the right plot. The right plot shows the intensity as function of the exit width (other widths fixed) and is used to select the exit width of $11 \mathrm{~cm}$. Although the positions of the maxima for the different reference experiments are quite different, for the selected compromise the intensities for all reference experiments are above $80 \%$ of the respective maximum. This best compromise can be different if one wants to optimise for a certain class of experiments. Note that neither the flux density nor integral flux at the guide exit are good criteria for this optimisation (the flux density can be maximised by minimising the exit width, resulting in a large divergence, and the integral flux by a wider guide, resulting in reduced flux density) and are therefore not plotted.

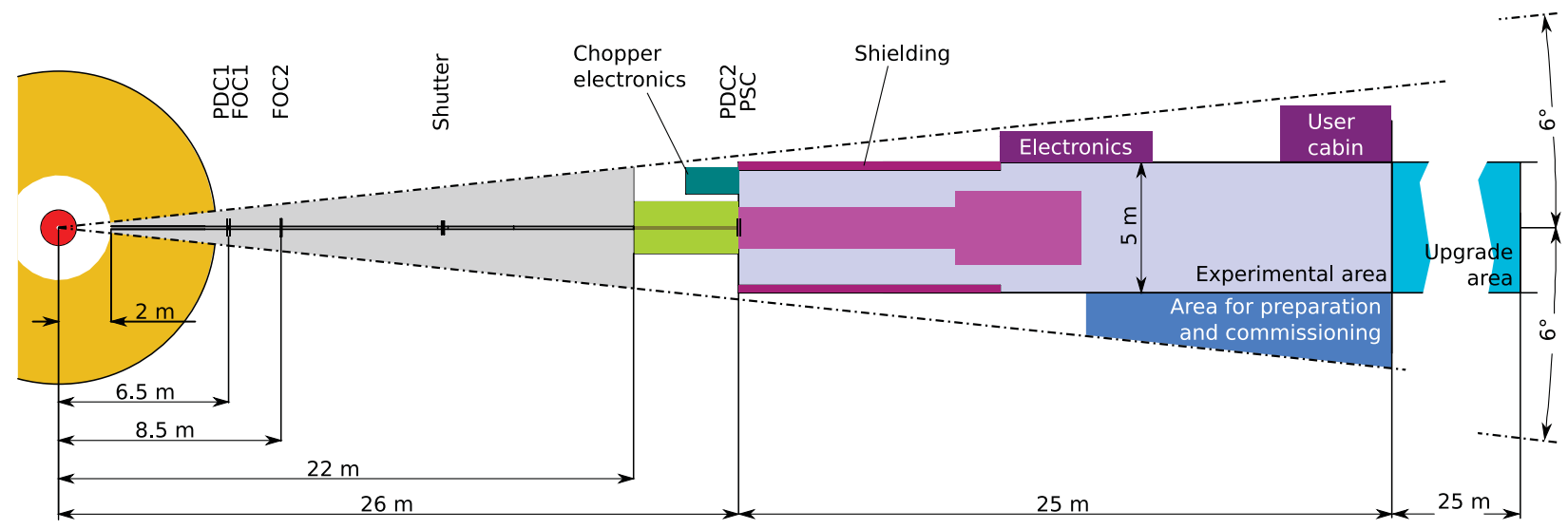

Figure 3. Proposed floor plan of the ANNI facility (schematic). Standard colors are as in Fig. 1. To give a scale, the magenta area indicates a PERC-like instrument with secondary spectrometer in measuring position. The upgrade area is needed for long experiments such as BeamEDM.

\subsection{Chopper system}

The chopper system consists of two frame overlap choppers (FOC1, FOC2), two pulse-defining choppers (PDC1, PDC2) and one pulse-suppressing chopper PSC. The PDCs are composed of pairs of co-rotating disks in order to adapt the opening angle to the experiment. Running the PDCs at multiples of the ESS repetition rate $14 \mathrm{~Hz}$ (e.g. at $70 \mathrm{~Hz}$ ) and using two counter-rotating pairs instead of one single pair per PDC greatly increases the rate in the experiments since chopper transition times (time intervals of only partly opened beam during the passage of a chopper slit) are strongly reduced and the chopper openings can be larger for a given pulse localisation [29]. The PSC is needed to suppress the higher order pulses of the PDCs.

Pulse multiplication can be applied at ANNI by replacing the PDC2 by a PMC, which is a multi slit chopper with slits at different angular distances from each other and of different sizes. The additional pulses appear at different neutron wavelengths which enables certain systematic checks for each source pulse. Since this PMC has to rotate at the ESS repetition rate, two counter-rotating disks are necessary in order to obtain acceptable chopper transition times. The layout of the PMC has to be adapted to the requirements of the specific experiment. Studies for PERKEO III indicate that increasing the rotation frequency of the standard PDCs (with adapted slit sizes) brings a higher gain in statistics than pulse multiplication [29], however, the benefit of pulse multiplication strongly depends on the experiment.

The parameters of the choppers are summarised in Table 2. The following chopper modes are available:

Full intensity: All choppers in rest in open position. Frame overlap occurs.

Maximum intensity with wavelength information: Only FOC1 and FOC2 are running and prevent frame overlap up to $130 \AA$, simultaneously selecting a wavelength band of $6 \AA$ width. The position of the 
Table 2. Key parameters of the choppers. Dist: Distance from the moderator, Rad: Radius of the chopper disk (indicative). Freq: Rotation frequency range (used frequencies are multiples of $14 \mathrm{~Hz}$ ). Angle: Opening angle of the chopper slit. X-section: Beam cross-section at the position of the chopper. Choppers are placed left or right of the beam to minimise the transition times. (E) indicates chopper disks that shall be compatible with a close-by particle physics experiment (non-depolarising, compatible with high vacuum, coated with ${ }^{6} \mathrm{Li}$ for low gamma background).

\begin{tabular}{lcccccl}
\hline Chopper & $\begin{array}{c}\text { Dist } \\
{[\mathrm{m}]}\end{array}$ & $\begin{array}{c}\mathrm{Rad} \\
{[\mathrm{cm}]}\end{array}$ & $\begin{array}{c}\text { Freq } \\
{[\mathrm{Hz}]}\end{array}$ & $\begin{array}{c}\text { Angle } \\
{\left[{ }^{\circ}\right]}\end{array}$ & $\begin{array}{c}\text { X-section } \\
{\left[\mathrm{cm}^{2}\right]}\end{array}$ & Comment \\
\hline PDC1 & 6.5 & 40 & $14-70$ & Variable & $13 \times 6$ & 2 counter-rotating pairs of co-rotating disks \\
FOC1 & 6.5 & 40 & 14 & 64 & $13 \times 6$ & Single disk \\
FOC2 & 8.5 & 40 & 14 & 79 & $13 \times 6$ & Single disk \\
PDC2 (E) & 26 & 40 & $14-70$ & Variable & $11 \times 7$ & 2 counter-rotating pairs of co-rotating disks \\
PSC (E) & 26 & 40 & 14 & 60 & $11 \times 7$ & Single disk, for PDC2 frequencies $>14 \mathrm{~Hz}$ \\
PMC (E) & 26 & 40 & 14 & Multi slit & $11 \times 7$ & 2 counter-rotating disks, replaces PDC2 \\
\hline
\end{tabular}

wavelength band can be chosen by changing the phases of both FOCs.

Localisation in time: Frame overlap is suppressed by the FOCs. PDC2 is running with large opening adjusted to the highest intensity of the beam. Depending on the wavelength bandwidth, PDC2 is run at a high frequency, in combination with the PSC.

Monochromatic: All choppers are phased to wavelength $\lambda_{0}$. The PDCs run at high frequency, in combination with the PSC. The opening angles of the PDCs are tuned to let all neutrons of wavelength $\lambda_{0}$ pass.

Localisation in space: As Monochromatic mode, but the opening angles and phases of the PDCs are tuned to the dimensions of a spectrometer such as PERC or PERKEO III (see $[16,29]$ and appendix A).

\subsection{Polarising options}

Neutron beam polarisation is necessary for many particle physics experiments where the requirements depend on the experiment: Searches for non-zero values of small asymmetries require highest intensity and can trade in a larger uncertainty of neutron beam polarisation, whereas accurate absolute measurements of asymmetries start to need $10^{-4}$ accuracy on the polarisation value $[15,30]$. Therefore ANNI provides different options for neutron polarisation:

Moderate polarisation at highest intensity: Bender 2 is replaced by a polarising supermirror bender of identical dimensions. The only additional losses of beam intensity by the polariser are due to the polarisation process itself and potentially due to lower reflectivities of polarising supermirrors compared to standard supermirrors.

Highest polarisation: The polarising supermirror bender (see previous point) is combined with a second polarising supermirror bender in the beam definition area. The two polarising benders implement the X-SM geometry [31]. The neutron spin is rotated adiabatically between the two polarisers. For this geometry, an average beam polarisation of well above $99.9 \%$ can be expected since the second polariser can be compact (e.g. solid state polariser [32]) and be installed in a high magnetising field, suppressing depolarisation by the supermirror [33]. In general the beam will be deflected horizontally by the second polariser which necessitates a lateral translation of the PDC2.

Polarisation with analytical wavelength dependence: For pulsed beams, the analytical wavelength dependence of the beam polarisation after a ${ }^{3} \mathrm{He}$ spin filter can be exploited directly for precision neutron polarimetry [7]. The guide is used in its non-polarising configuration with Bender 2. A ${ }^{3} \mathrm{He}$ spin filter cell is installed in the beam definition area, either a MEOP cell which can already stand the neutron intensity or a SEOP cell if the performance losses in high flux [34] can be compensated. An adiabatic fast passage spin flipper for ${ }^{3} \mathrm{He}$ allows inverting the spin direction insitu which provides possibilities for systematic checks. Spin flipping: An adiabatic fast passage spin flipper for neutrons or other flipper types can be installed in the beam definition area.

For polarisation analysis, opaque ${ }^{3} \mathrm{He}$ spin filter cells in a magnetic environment with intrinsic ${ }^{3} \mathrm{He}$ spin flipping shall be available, enabling $10^{-4}$ accuracy $[33,35]$. The preferred method for polarising these cells is a central MEOP facility at the ESS, similar to the Tyrex facility at the ILL $[36,37]$.

\section{Expected performance}

The simulated divergence distributions in horizontal and vertical direction and capture flux density spectrum at the guide exit of ANNI are shown in Fig. 4. The simulated capture flux density averaged over the guide exit of $11 \times 7 \mathrm{~cm}^{2}$ and time is $2.0 \times 10^{10} \mathrm{n} /\left(\mathrm{cm}^{2} \mathrm{~s}\right)$ for the wavelength band $2 \AA$ to $8 \AA$ (selected by the FOCs) and $2.5 \times 10^{10} \mathrm{n} /\left(\mathrm{cm}^{2} \mathrm{~s}\right)$ for the full spectrum (all choppers stopped in open position), comparable to the capture flux density at the guide exit of the instrument PF1B [38] at the ILL $\left(2.2 \times 10^{10} \mathrm{n} /\left(\mathrm{cm}^{2} \mathrm{~s}\right)\right)$. The simulated particle flux density at $8.9 \AA$, the wavelength relevant for singlephonon UCN production in He UCN sources [39], is $2.1 \times$ $10^{8} \mathrm{n} /\left(\mathrm{cm}^{2} \mathrm{~s} \AA\right)$. At ANNI, polarised fluxes for the option of moderate polarisation are only a factor 2 lower (see Sect. 3.5 ), whereas geometrical and transmission losses of the polarising bender (typically another factor of 2) have to be taken into account at other facilities.

The expected gain factors for the reference experiments are given in Table 3. 

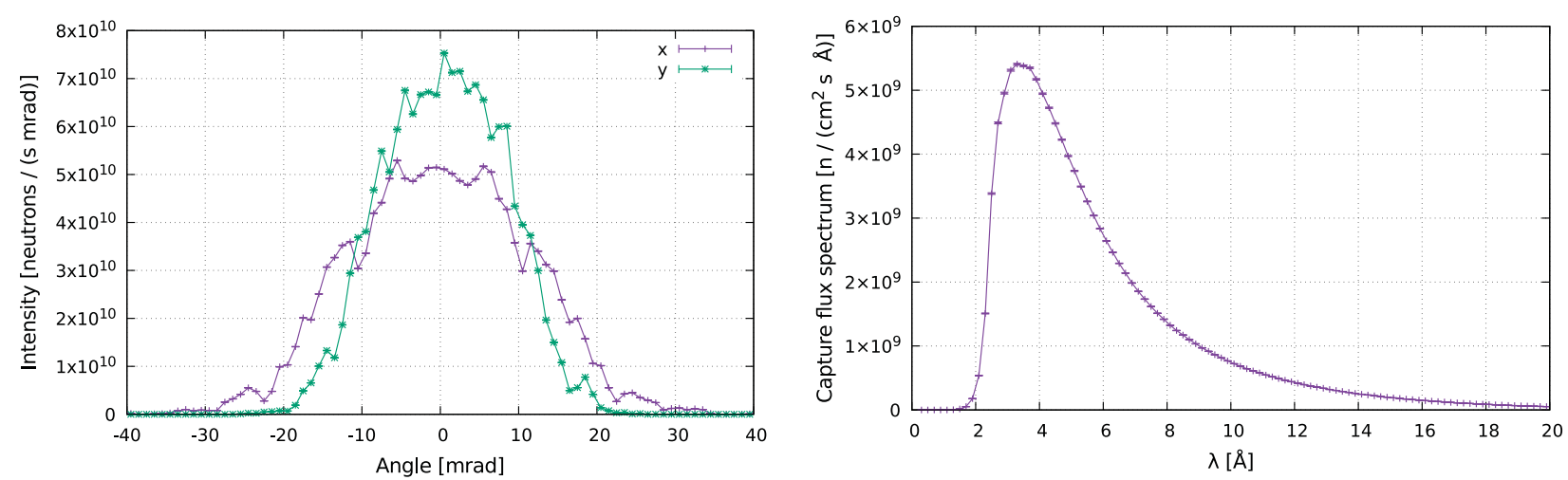

Figure 4. Left: Simulated divergence distributions in horizontal $(x)$ and vertical $(y)$ direction at the guide exit for neutrons between $2 \AA$ and $8 \AA$ (weighted with their capture cross-section and integrated over the other direction and the guide exit area). Right: Simulated capture flux density spectrum averaged over the guide exit.

Table 3. Simulated gain in counting rate for the reference experiments at ANNI (ESS at $5 \mathrm{MW}$ ) relative to the respective presently used facility. Gains marked by ${ }^{\uparrow}$ refer to polarised beams. Details on the determination of the gain factors are given in appendix A.

\begin{tabular}{lcl}
\hline Experiment & Gain & Comment \\
\hline aSPECT & 1.3 & Full spectrum \\
& 2.8 & $\begin{array}{l}\text { Localisation in time to } 1 / 3 \text { of } \\
\text { ESS period }\end{array}$ \\
NPDGamma & $27^{\uparrow}$ & Wavelength information \\
PERC & $15^{\uparrow}$ & Localisation in space \\
PERKEO III & $17^{\uparrow}$ & Localisation in space \\
\hline
\end{tabular}

\section{Conclusions and outlook}

The proposed neutron beam facility ANNI will provide unprecedented pulsed intensity for particle physics experiments and its time-averaged flux will still at least equal existing reactor facilities. Its design allows to fully exploit the ESS pulse structure and to use the optimum polarisation option for each experiment. Particle physics experiments using pulsed beams will gain one order of magnitude in event rate compared to other facilities. ANNI will enable a new level of accuracy in measurements of correlation coefficients in neutron beta decay, novel methods to determine electromagnetic properties of the neutron and systematic access to the tiny effects of hadronic weak interaction in calculable systems.

The design proposed in this paper can be further optimised and should therefore be considered as preliminary. In particular, particle physics experiments have more demanding background requirements than instruments for neutron scattering. Therefore the ANNI design should be validated and optimised for background suppression from the spallation source. As example, the long bending sections may be replaced by compact solid state benders, which may result in more localised sources for secondary particles that are easier to shield. This would also allow for a compact solid state polariser [32] as integrated polariser option with better performance. Furthermore, the design proposed here is chosen as best compromise for the considered suite of reference experiments but scientific priorities could also call for full optimisation towards one specific experiment.

We thank Dr. Grammer and Dr. Fomin for sharing the MCNPX model of NPDGamma at the SNS and Mr. Hollering for providing McStas parametrisations of the supermirrors of the PERC internal guide.

\section{A. Reference experiments}

The suite of reference experiments was selected in order to cover most of the particle physics experiments at cold neutron beams. A short description of the selected experiments is given below; the most relevant parameters for the simulations are summarised in Table 4.

aSPECT $[18,19]$ is the prototype for a neutron decay experiment with short decay volume, low sensitivity to gamma background and consequently a large angular acceptance for neutrons. The experiment uses an unpolarised continuous beam. Since Penning traps may cause background with time constants large compared to the ESS pulse length [40], aSPECT may profit from localisation of the neutron pulse in time for an improved signal-to-background ratio. From the collimation system used in the aSPECT experiment (see [41]), only the last aperture in front of the spectrometer and all apertures inside the cryostat were used in the simulations since this is preferable for statistics and systematics (smaller edge effect because of the more homogeneous beam profile). The gain in Table 3 corresponds to the ratio of the simulated capture intensities in the decay volume for aSPECT at ANNI (all choppers at rest, full neutron spectrum) and at PF1B. Pulse localisation in time was realised by the PDC2 chopper directly upstream of the last aperture in front of the spectrometer. In the simulations of PF1B, only this chopper was used; in case of ANNI additionally the FOCs suppressed frame overlap. The PDC2 was run at $14 \mathrm{~Hz}$ and its opening was chosen to limit the arrival of neutrons in the decay volume to $1 / 3$ of the chopper period (at PF1B, $10^{-4}$ of the intensity were allowed in the other $2 / 3$ since a single chopper does not cut the tail of the spectrum). This time localisation reduces the time-averaged counting rate at ANNI to about $62 \%$ and at PF1B to about $28 \%$ of the respective full rate. 
Table 4. Parameters of the reference experiments used in the simulations. The extension guide bridges the distance between the exit of the ANNI guide and the start of the collimation system, in order to place the experiment itself at the start of the experimental area, and has the same cross-section and $m$ value as the ANNI guide. For important apertures the distance to the end of the extension guide and the cross-section are given. Criterion indicates the quantity that was used for optimisation; it is measured after the last aperture. The PERC setup is too different and is described in the text.

\begin{tabular}{lllll}
\hline Experiment & aSPECT & NPDGamma & PERC & PERKEO III \\
\hline Wavelength range & $2.0-8.0 \AA$ & $3.1-9.1 \AA$ & $4.5-5.5 \AA$ & $4.5-5.5 \AA$ \\
Extension guide & $1.5 \mathrm{~m}$ & $3.1 \mathrm{~m}$ & see text & $0.55 \mathrm{~m}$ \\
First aperture & as guide exit & as guide exit & see text & $6 \times 6 \mathrm{~cm}^{2}$ \\
Defining apertures & $2.484 \mathrm{~m}: 4.5 \times 7.0 \mathrm{~cm}^{2}$ & $0.92 \mathrm{~m}: 16 \times 16 \mathrm{~cm}^{2}$ & see text & $3.23 \mathrm{~m}: 6 \times 6 \mathrm{~cm}^{2}$ \\
& $2.894 \mathrm{~m}: 4.5 \times 7.0 \mathrm{~cm}^{2}$ & $1.72 \mathrm{~m}: 18 \times 22 \mathrm{~cm}^{2} \cap \varnothing 22 \mathrm{~cm}$ & & \\
Criterion & Capture flux & Particle flux & see text & Capture flux \\
\hline
\end{tabular}

NPDGamma $[20,21]$ is the prototype for target experiments with large target size, large divergence acceptance and beam polarisation optimised for intensity. Frame overlap suppression is used. The parameters of the collimation system were extracted from the MCNPX model [42]. Since 43\% of the neutrons incident on the target are captured by hydrogen [21], the particle flux is used as optimisation criterion. The gain in Table 3 corresponds to the ratio of the integral polarised particle flux at the target position without frame overlap for ANNI (polarisation by polarising bender 2 implemented in the guide, FOCs set to 3.1-9.1 $\AA$ in order to use the same lower wavelength limit as the experiment, chosen to remain below the $14.7 \mathrm{meV}$ threshold for spinflip scattering, see [43]) and FnPB [44] (3.1-6.6 ̊, flux from [21] scaled to 1.4 MW nominal power of the Spallation Neutron Source SNS). The larger wavelength band at ANNI in spite of the longer guide is enabled by the lower repetition rate of the ESS $(14 \mathrm{~Hz})$ compared to the SNS $(60 \mathrm{~Hz})$.

PERC [15] is the prototype for experiments where the fiducial volume is (almost) directly coupled to the primary neutron guide and where the divergence acceptance corresponds to that of a neutron guide. The fiducial volume of PERC consists of an $8 \mathrm{~m}$ long internal neutron guide where charged neutron decay products are extracted by a strong longitudinal magnetic field. The experiment will profit from pulse localisation in space in order to avoid regions of illdefined spectrometer response. The beam configuration and the parameters of the internal guide were extracted from a McStas model [45]. For the ANNI simulations, PERC is placed such that the chopper position corresponds to that of PDC2. The internal guide must be non-depolarising at the $10^{-4}$ level which is why CuTi supermirrors are used, with $m=1.95$. The beam preparation area is bridged by an $m=1.8$ guide of $7.0 \times 6.0 \mathrm{~cm}^{2}$ and $4 \mathrm{~m}$ length (the lower $m$ value is chosen in order to reduce divergence losses inside PERC), followed by an $80 \mathrm{~cm} m=1.95 \mathrm{CuTi}$ guide after a $20 \mathrm{~cm}$ gap for the PDC2. The internal guide starts after a gap of $50 \mathrm{~cm}$ (needed for a backscattering detector for charged particles). It has $6 \mathrm{~cm}$ height and consists of through-going top and bottom plates and 4 sections of $1.98 \mathrm{~m}$ long side plates with $2 \mathrm{~cm}$ pumping gap and widths of $7.0 \mathrm{~cm}, 7.2 \mathrm{~cm}, 7.4 \mathrm{~cm}$, and $7.6 \mathrm{~cm}$, respectively. The intensity was determined by averaging the capture flux over squares of $5.0 \times$ $5.0 \mathrm{~cm}^{2}$ between the four sections (the smaller area accounts for the fact that decay particles close to the guide surfaces are lost by gyration). The guide optimisation was done for a limited wavelength band as needed for pulse localisation in space.

Perkeo III [22] is the prototype for experiments with a long decay volume and limited angular acceptance (about $10 \mathrm{mrad}$ ) for neutrons. The experiment uses pulse localisation in space in order to single out decay events in a region with well-defined spectrometer response and free from beam-related background. The collimation system was taken from [46] and placed such that the chopper position corresponds to that of PDC2. A limited wavelength band was used for the guide optimisation in order to simulate pulse localisation in space.

The gains for PERC and PERKeO III in Table 3 are defined as the ratio of the time-averaged decay rates at ANNI and at PF1B [38] for the neutron pulse fully contained in the homogeneous part of the decay volume (pulse localisation in space). At PF1B, an estimated geometrical and transmission loss of 50\% by the polarising bender was taken into account; this loss is included in the simulations for ANNI with polarising bender 2 implemented in the guide (the factor $1 / 2$ for spin selection is the same in both cases). At ANNI, pulse localisation was achieved by running both PDCs at $70 \mathrm{~Hz}$; the other parameters of the PDCs were optimised for maximum count rate. FOCs and PSC were used to suppress unwanted pulses. At PF1B, pulse localisation was achieved by combining a Dornier velocity selector [47] with a chopper. For PERKEO III the single disk chopper used in the experiment was simulated (see [46]); the resulting decay rate agreed with the experimental one. For PERC a pair of counter-rotating disk choppers was used; selector rotation frequency, chopper slit size and chopper frequency were optimised for maximum time-averaged decay rate with the condition of at least $2 \mathrm{~ms}$ for background measurement between subsequent pulses, without neutrons between chopper and beam stop (at ANNI this condition is fulfilled due to the low ESS repetition rate). The statistically optimal neutron pulse length at the end of the PERC decay volume was found to be $5 \mathrm{~m}$ for both ANNI and PF1B. The performance of the new dedicated 
PERC beam line MEPHISTO [48] at the FRM-II is expected to be similar to PF1B. The expected gains for unpolarised measurements would be a factor 2 lower and are comparable to the simplified estimates in [16]. Further details on the simulations can be found in [29] (where slightly smaller horizontal dimensions of the ANNI guide were used).

\section{References}

[1] H. Abele, Prog. Part. Nucl. Phys. 60, 1 (2008)

[2] D. Dubbers, M.G. Schmidt, Rev. Mod. Phys. 83, $1111(2011)$

[3] V. Cirigliano et al., Prog. Part. Nucl. Phys. 71, 93 (2013)

[4] R. Gupta et al., Phys. Rev. D 98, 034503 (2018)

[5] M. Gonzalez-Alonso et al., Prog. Part. Nucl. Phys. 104, 165 (2019)

[6] T. Chupp et al., Rev. Mod. Phys. 91, 015001 (2019)

[7] S.I. Pentilla, J.D. Bowman, J. Res. Natl. Inst. Stand. Technol. 110, 309 (2005)

[8] F.M. Piegsa, Phys. Rev. C 88, 045502 (2013)

[9] F.M. Piegsa, Phys. Rev. C 98, 045503 (2018)

[10] R. Maruyama et al., Phys. B 335, 238 (2003); Nucl. Instr. Meth. A 530, 505 (2004)

[11] B. Märkisch, Phys. Proc. 51, 37 (2014)

[12] K.H. Andersen et al., J. Appl. Cryst. 51, 264 (2018)

[13] C. Theroine et al., ANNI - a cold neutron beam facility for Particle Physics, ESS Instrument construction proposal to 2015 Round, unpublished (2015)

[14] S. Gardner et al., Ann. Rev. Nucl. Part. Scien. 67, 69 (2017)

[15] D. Dubbers et al., Nucl. Instr. Meth. A 596, 238 (2008)

[16] C. Klauser et al., Phys. Proc. 51, 46 (2014)

[17] D. Dubbers, Nucl. Instr. Meth. A 349, 302 (1994)

[18] O. Zimmer et al., Nucl. Instr. Meth. A 440, 548 (2000)

[19] F. Glück et al., Eur. Phys. J. A 23, 135 (2005)

[20] M.T. Gericke et al., Phys. Rev. C 83, 015505 (2011)

[21] D. Blyth et al., Phys. Rev. Lett. 121, 242002 (2018)

[22] B. Märkisch et al., Nucl. Instr. Meth. A 611, 216 (2009)

[23] K. Bodek et al., EPJ Web of Conf. 219, 04001 (2019)

[24] A. Young, J. Barrow, EPJ Web of Conf. 219, 07005 (2019)
[25] L. Zanini et al., Moderator Configuration Options for ESS, in T. Oku et al. (eds.), ICANS XXI (JAEA-Conf 2015-002, DOI:10.11484/jaea-conf-2015-002, 2015) $126-133$

[26] P. Willendrup et al., Nucl. Instr. Meth. A 634, 150 (2011); http: //www.mcstas.org/

[27] K. Andersen, Report ESS-0080327 Rev 2, European Spallation Source (2016)

[28] T. Soldner et al., arXiv:1811.11692, ANNI McStas file included as ancillary file (2019)

[29] P. Torres-Sánchez, Optimization of the cold neutron beamline ANNI, Internship report, Institut LaueLangevin (2018); Master thesis (TFM), Universidad de Granada (2018)

[30] B. Märkisch et al., Phys. Rev. Lett. 122, 242501 (2019)

[31] M. Kreuz et al., Nucl. Instr. Meth. A 547, 583 (2005)

[32] A.K. Petukhov et al., Nucl. Instr. Meth. A 838, 33 (2016)

[33] C. Klauser et al., Nucl. Instr. Meth. A 840, 181 (2016)

[34] M. Sharma et al., Phys. Rev. Lett. 101, 083002 (2008)

[35] T. Soldner et al., Experimental report 3-07-235, Institut Laue-Langevin (2011)

[36] K.H. Andersen, Physica B 356, 103 (2005)

[37] A.K. Petoukhov, Phys. B 385-86, 1146 (2006)

[38] H. Abele et al., Nucl. Instr. Meth. A 562, 407 (2006)

[39] R. Golub, J.M. Pendlebury, Phys. Lett. A 62, 337 (1977)

[40] R. Maisonobe, $\mathrm{PhD}$ thesis, Université de Grenoble (2014), https://tel.archives-ouvertes.fr/ tel-01060163

[41] M. Borg, PhD thesis, Universität Mainz (2010), http://nbn-resolving.org/urn:nbn:de: hebis : 77-26691

[42] K.B. Grammer, N. Fomin, private communication (2017)

[43] K.B. Grammer et al., Phys. Rev. B 91, 180301(R) (2015)

[44] N. Fomin et al., Nucl. Instr. Meth. A 773, 45 (2015)

[45] A. Hollering, B. Märkisch, private communication (2017)

[46] H.F. Mest, PhD thesis, Universität Heidelberg (2011), doi:10.11588/heidok.00012198

[47] H. Friedrich et al., Phys. B 156\&157, 547 (1989)

[48] Heinz Maier-Leibnitz Zentrum et al., J. Large-Scale Res. Facil. 1, A21 (2015) 